\title{
Étude des caractères d'enracinement et de leur rôle dans l'adaptation au déficit hydrique chez l'orge (Hordeum vulgare $L$ )
}

\author{
A Khaldoun *, J Chery, P Monneveux \\ Chaire de Phytotechnie et d'amélioration des plantes (ENSAM), 2, place Viala, 34060 Montpellier Cedex 01; \\ INRA, station de génétique et d'amélioration des plantes, Domaine de Melgueil, 34130 Mauguio, France
}

(Reçu le 2 novembre 1989; accepté le 18 mars 1990)

\begin{abstract}
Résumé - Les effets d'un déficit hydrique sur les caractéristiques de l'enracinement de 5 variétés d'orge (Hordeum vulgare $L$ ) ont été comparés. Les génotypes, originaires de la zone méditerranéenne, ont été cultivés en serre et en pots sous différentes conditions de déficit hydrique. Cela a permis de mettre en évidence d'importantes différences variétales pour la plupart des caractères étudiés :

- nombre de racines principales;

- volume racinaire;

- rapport entre la masse de matière sèche des parties aériennes et la masse de substance sèche des racines;

- surface foliaire et potentiel hydrique ( $\psi f$ ) de la dernière feuille (stade B2 de Jonard), exprimé en MPa.

Par contre pour le caractère masse de matière sèche du système racinaire, les différences ne sont pas significatives. Le rôle de ces caractéristiques d'enracinement dans la tolérance au déficit hydrique est discuté; les résultats enregistrés sur les différentes variétés sont rapprochés de ceux obtenus au champ, dans les conditions diverses d'alimentation hydrique.
\end{abstract}

déficit hydrique / tolérance à la sécheresse / système racinaire / Hordeum vulgare L / potentiel hydrique / variété

Summary - Effects of drought stress on root characteristics of five barley (Hordeum vulgare $\mathrm{L}$ ) varieties. The effects of water deficit on root characteristics of 5 barley (Hordeum vulgare L) varieties were compared. These genotypes from the Mediterranean region were cultivated in pots in a greenhouse, under irrigated conditions and water deficit. Important varietal differences were observed for:

- number of primary roots;

- root volume;

- shoot/root ratio;

- leaf water potential ( $\psi f$ ) expressed in MPa.

There was no significant difference between the root system dry matter in both treatments. The role of the root characteristics in water deficit tolerance and their relation with some agronomical characters are discussed; the root development seems to be closely related to stem height, and earliness, and plays a relevant role in drought tolerance; particularly in the case of severe water stress: the Algerian variety Tichedrett, with its deep and well ramified root system grows well in very dry conditions; this is demonstrated by field data obtained in Setif (Algeria). In the case of moderate and terminal water stress (the most frequent in the South of France), high yields seem to be obtained through a high productivity and a good earliness (varieties Barberousse (France) and Faiz (ICARDA). Because of the diversity of combinations between phenological and morphological parameters of adaptation, it is necessary, before selecting for drought tolerance to know the water stress conditions and objectives.

drought stress / drought tolerance / root system / Hordeum vulgare $L$ / water potential / variety

\footnotetext{
*Adresse actuelle : Institut technique des Grandes cultures, département recherche, BP 16 El Harrach, Alger, Algérie.
} 


\section{INTRODUCTION}

Dans le monde, presque $40 \%$ des terres cultivables sont touchées chaque année par la sécheresse (Oertli, 1983), ce qui réduit considérablement le rendement des cultures. Le transfert de l'eau du sol vers les parties aériennes via les racines joue un grand rôle dans la réponse des plantes à la sécheresse.

Pour réduire les effets de la sécheresse, la plante a 2 possibilités : réduire ses pertes d'eau interne par transpiration et-ou maintenir une meilleure alimentation hydrique malgré une réduction des disponibilités en eau du milieu.

La profondeur et la densité racinaires jouent, d'après Ahmadi (1983), un rôle important dans le maintien de l'approvisionnement en eau d'une plante soumise à la sécheresse. Certes, les caractéristiques du système racinaire varient fortement avec les conditions édaphiques et climatiques (Baldy, 1973; Brown et al, 1987; Souty, 1987) : la croissance des racines est, par exemple, souvent gênée par des obstacles (semelle de labour ou couche de tuf) et elle dépend étroitement de l'état hydrique du sol (Lomas et Shashoua, 1973). Mais ces caractéristiques d'enracinement sont liées également au génotype : pour de nombreux auteurs (Hurd, 1974, 1976; Burch et al, 1978; O'Brien, 1979; Passioura, 1981), le développement racinaire est considéré comme l'un des facteurs déterminants de la variabilité inter- et intraspécifique concernant la tolérance à la sécheresse du blé; Ali-Zade et Sultanov (1970) mettent en évidence de grandes variations dans les génotypes caractérisant la profondeur d'enracinement pour un même état hydrique du sol. Les travaux de Brown et al (1987) sur l'orge, menés en serre, révèlent également des différences intervariétales. Hurd (1974 et 1976), Quizenberry (1982), Malet (1971) et Sullivan (1983) établissent une corrélation positive, en conditions sèches, entre le rendement et le développement racinaire de différentes espèces (orge, blés, sorgho). Bierhuissen (1974 et 1981), et Jordan et Miller (1980) observent une relation entre les valeurs élevées du rapport masse de matière sèche des parties aériennes sur masse de matière sèche des racines, et la sensibilité à la sécheresse.

Certaines caractéristiques d'enracinement paraissent très liées à certains facteurs agronomiques comme l'aptitude au tallage (Black, 1970; Masle, 1980), la précocité (Grignac, 1987), l'alternativité (Janjatovic et al cités par Baldy, 1973) et la hauteur du chaume. En ce qui concerne ce dernier point, les travaux de Subbiah et al (1968), Hurd (1974), Bagga et al (1970) sur le blé montrent que certains gènes de nanisme sont associés à une réduction du système racinaire. Pour l'orge, cette relation entre nanisme et développement racinaire est parfois controversée : Ali (1977) constate par exemple un enracinement plus développé chez les variétés deminaines; par contre, Irvine et al (1980) n'observent aucune différence significative d'enracinement entre des variétés demi-naines et des variétés à paille haute, cultivées à différents niveaux de déficit hydrique.

Le but de notre étude a été :

- de vérifier l'existence d'une variabilité à l'intérieur de l'espèce orge (Hordeum vulgare $\mathrm{L}$ ) pour différentes caractéristiques de l'enracinement;

- de juger, chez les variétés étudiées, les effets du déficit hydrique sur ces caractéristiques, ainsi que sur l'état de l'eau dans la feuille (caractérisé par le potentiel hydrique foliaire) et sur la croissance (estimée par la surface de la dernière feuille, mesurée au stade B2, selon Jonard et al, 1952).

\section{MATÉRIEL ET MÉTHODES}

L'étude porte sur 5 cultivars d'orge, originaires de 3 régions du Bassin méditerranéen :

- Tichedrett, orge à 6 rangs sélectionnée dans une population originaire des hauts plateaux algériens, peu productive, mais tolérante à des déficits hydriques très intenses;

- Barberousse, orge à 6 rangs, cultivée en France et en Afrique du Nord;

- Matnan et Rihane, orges à 6 rangs sélectionnées par l'ICARDA *, Syrie;

- Faiz (en arabe : la gagnante) variété à 2 rangs (Syrie, Tunisie).

Quelques caractéristiques agronomiques de ces variétés sont rapportées dans le tableau I.

\section{Dispositif expérimental}

Les grains d'orge sont mis à germer en boîte de Petri; les plantules sont repiquées dans des pots en polyéthylène de 8,3 I (17 $\times 17 \times 24 \mathrm{~cm})$, à raison de 2 plantules par pot, dans un sol reconstitué avec un mélange de terre (argilo-limoneuse), de sable et de

\footnotetext{
* International Center for Agricultural Research in Dry Areas, Alep, Syria.
} 
Tableau I. Quelques caractéristiques morpho-physiologiques des 5 variétés d'orge cultivée, utilisées dans l'étude; moyenne des résultats d'essais obtenus sur 2 années de culture à la station d'amélioration des plantes de Melgueil (INRA-Montpellier et Sétif (Algérie) 1978/1979 et 1987/1988. La précocité est donnée par le nombre de jours compris

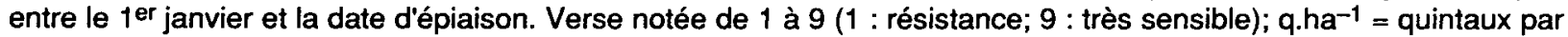
hectare; I = irrigué; $\mathrm{NI}=$ non irrigué; * = effet important de la verse; les chiffres entre parenthèses représentent le classement; $N U I=$ Indice de sensibilité au déficit hydrique.

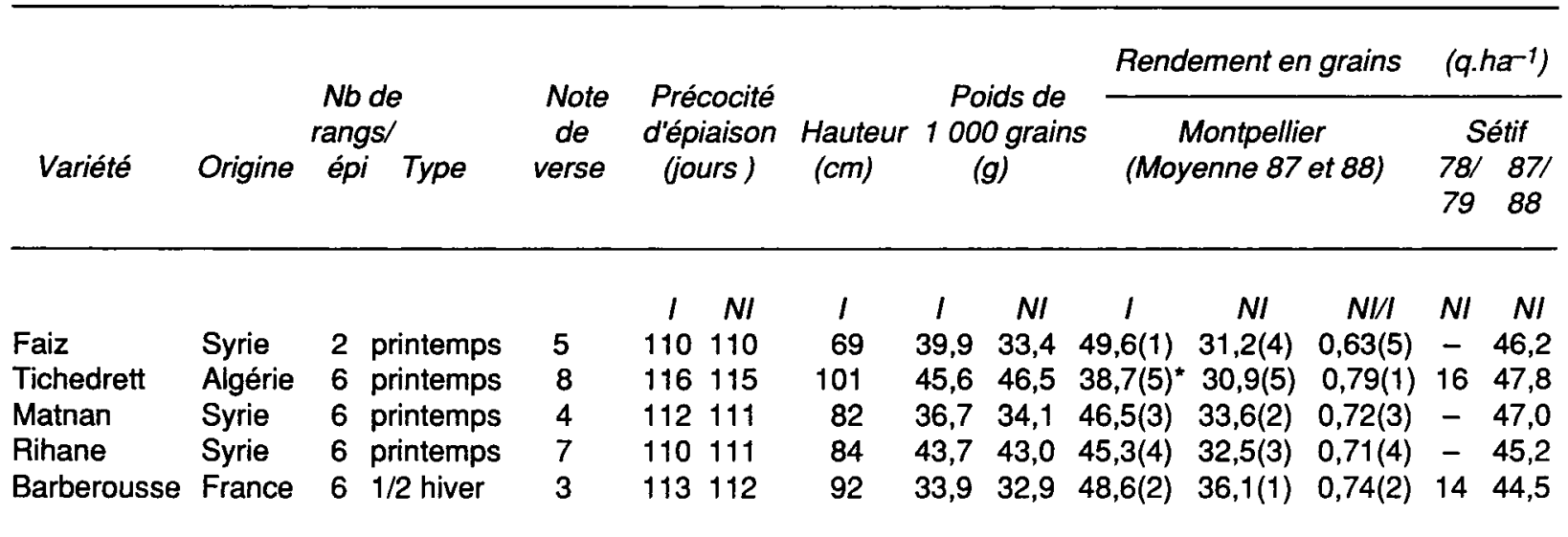

terreau (en proportion $1: 8: 1$ ), de faible capacité de rétention ( $16 \%$ d'humidité).

L'expérimentation est menée en serre climatisée (température de $20^{\circ} \mathrm{C}$ le jour, de $15^{\circ} \mathrm{C}$ la nuit, humidité relative variant entre 70 et $80 \%$, intensité lumineuse moyenne $85 \mathrm{~W} \cdot \mathrm{m}^{-2}$, photopériode $15 \mathrm{~h}$ ). L'essai, disposé en forme de split-plot, comporte 2 lots (traitement avec ou sans irrigation); chaque lot est composé de 5 variétés, répétées 4 fois.

Les 2 lots ont été irrigués, à la capacité au champ avec de l'eau du robinet jusqu'à la levée, puis avec une solution nutritive complète jusqu'au stade 2 à 3 feuilles, les régimes d'irrigation sont ensuite modifiés; dans le cas du lot témoin, les pots sont maintenus constamment humides avec une dose de $120 \mathrm{ml}$ d'eau à chaque apport, alors que dans le cas des plantes soumises au déficit hydrique, la même quantité d'eau n'est apportée que lorsque les plantes atteignent le point de flétrissement, correspondant à un potentiel foliaire $(\psi f)$ d'environ 2,5 MPa.

\section{Mesures}

Le prélèvement des échantillons est effectué au stade montaison (stade B2 de Jonard). Les pots sont soigneusement vidés de leur contenu; le système racinaire est alors trempé dans un bac rempli d'eau du robinet pour un premier lavage, puis plongé dans un autre bac d'eau à laquelle est ajouté du détartrant (Calgon) à une dose de $5 \mathrm{~g} / \mathrm{l}$, afin de désagréger les particules de matière organique collées aux racines; le système racinaire est ensuite mis à sécher entre 2 feuilles de papier.

Les mesures ont porté sur les caractéristiques d'enracinement suivantes:

— nombre de racines principales (NRP);
- nombre de racines de plus de $1 \mathrm{~cm}$ de long partant du plateau de tallage;

- longueur du système racinaire (LSR);

- volume racinaire sur 4 classes différentes de longueur racinaire $(A=0-10 \mathrm{~cm}, B=10-20 \mathrm{~cm}, C=20-$ $30 \mathrm{~cm}$ et $D \geq 30 \mathrm{~cm}$ ) le volume racinaire $(V R H)$ est mesuré par immersion, en appliquant la technique de Musick et al (1965);

- masse de matière sèche des racines par classe de longueur racinaire, obtenue après séchage à l'étuve à $80^{\circ} \mathrm{C}$ pendant $48 \mathrm{~h}$. Les longueurs racinaires supérieures à $24 \mathrm{~cm}$ (profondeur du pot) s'expliquent par le fait que les racines se sont enroulées au fond du pot.

Sur la partie aérienne, d'autres mesures ont été effectuées : nombre de talles par plante, masse de matière fraîche et de matière sèche de la partie aérienne (obtenue après séchage à l'étuve à $80^{\circ} \mathrm{C}$ pendant $48 \mathrm{~h})$.

La surface de la dernière feuille (au stade B2 de Jonard) est évaluée à l'aide d'un planimètre électronique (Lambda Ll-3 000), avant prélèvement de la plante. Le potentiel hydrique foliaire $(\psi t)$ est mesuré sur la même feuille à l'aide d'une presse à membrane du type de celle de Renard et Ndayishimie (1982). Les valeurs sont exprimées en MPa.

\section{RÉSULTATS}

L'étude statistique des données fait appel à la méthode d'analyse de la variance. Les différents. résultats obtenus permettent d'observer un effet variété hautement significatif $(P=0,0001)$ pour les variables suivantes : nombre de racines principales (NRP), longueur du système racinaire ( $L S R)$, volume total du système racinaire (VRH), 
et masse de matière sèche de la partie aérienne (MSP); l'effet variété est également significatif pour le nombre de talles par plante $(P=0,03)$. $P a r$ contre, les effets sur la masse de matière sèche racinaire et sur le rapport $Q$ (masse de matière sèche aérienne sur masse de matière sèche racinaire) ne sont pas significatifs.

L'analyse de la variance révèle également un effet significatif de l'alimentation hydrique, sauf sur la variable PMSSR (masse de matière sèche des racines).

L'interaction variété $x$ effet hydrique est hautement significative $(P=0,0001)$ pour les variables suivantes (tableau II) : longueur du système racinaire ( $L S R)$, nombre de racines principales $(N R P)$, volume total du système racinaire $(V R H)$, masse de matière sèche des parties aériennes (MSP) et rapport de la masse aérienne sur la masse racinaire (Q). Cela signifie donc que le classement variétal n'est pas le même pour ces variables en conditions de bonne alimentation et en conditions de limitation hydrique.

L'examen de la moyenne générale par traitement et des moyennes par génotype et par variable, classées à l'aide du test de Duncan dans les 2 traitements (avec et sans déficit hydrique), est consigné dans le tableau III.

\section{Nombre de racines principales}

Le nombre moyen de racines principales est fortement réduit chez tous les génotypes, lorsque l'on passe des plantes du lot témoin aux plantes soumises au déficit hydrique (tableau III). En condition de déficit hydrique, la variété Rihane possède le nombre de racines principales (primaires) le plus élevé, suivie des variétés Matnan, Barberousse et Faiz; la variété Tichedrett possède le nombre de racines primaires significativement le moins élevé. En absence de déficit hydrique (lot irrigué), les variétés se classent en 3 groupes :

- les variétés Tichedrett et Rihane;

- la variété Barberousse;

- les variétés Matnan et Faiz.

\section{Longueur de la racine principale}

La technique utilisée dans cette étude (pots de contenance limitée) n'a pas permis d'obtenir une expression réelle de l'enracinement profond. Des différences apparaissent toutefois entre les traitements $(13,1 \%$ entre le lot témoin et le lot sous contrainte hydrique) ainsi qu'entre génotypes (tableau III); en l'absence de déficit hydrique, la variété Tichedrett présente le système racinaire le plus profond, suivie de Faiz, Rihane et Matnan (pas de différences significatives entre ces 3 variétés), puis de la variété Barberousse; en conditions de déficit hydrique, la variété Tichedrett présente aussi le système racinaire le plus profond.

\section{Volume du système racinaire}

Le volume racinaire et la masse de matière sèche du système racinaire sont affectés par le

Tableau II. Analyse de variance, valeurs de $F$ calculé (Fisher) et de la probabilité de signification associée à la valeur de $F$, des caractères d'enracinement et de la partie aérienne. Effet variétal et hydrique. $C U \times H=$ cultivar $\times$ effet hydrique; $\mathrm{ns}=$ non significatif; $N R P=$ nombre de racines principales; $L S R=$ longueur du système racinaire; $V R H=$ volume du système racinaire; $N T=$ nombre de talles; $M S P=$ masse de la matìre sèche aérienne; $P M S S R=$ masse de la matière sèche racinaire; $Q=$ rapport entre masse aérienne et masse racinaire.

\begin{tabular}{|c|c|c|c|c|c|c|}
\hline \multirow{2}{*}{ Variable } & \multicolumn{2}{|c|}{ Effet variétal } & \multicolumn{2}{|c|}{ Effet hydrique } & \multicolumn{2}{|c|}{ Interaction $\mathrm{CU}^{*} \mathrm{H}$} \\
\hline & $F$ & $\operatorname{Pr}>f$ & $\mathrm{~F}$ & $\operatorname{Pr}>\mathrm{F}$ & $F$ & $\mathrm{Pr}>\mathrm{F}$ \\
\hline$N R P$ & 11,36 & 0,0001 & 337,9 & 0,0001 & 11,03 & 0,0001 \\
\hline$L S R$ & 29,57 & 0,0001 & 123,0 & 0,0001 & 12,14 & 0,0001 \\
\hline$V R H$ & 16,07 & 0,0001 & 198,51 & 0,0001 & 8,24 & 0,0001 \\
\hline$N T$ & 2,88 & 0,03 & 135,6 & 0,0001 & 2,37 & 0,06 \\
\hline$M S P$ & 6,90 & 0,0002 & 86,37 & 0,0001 & 13,50 & 0,0001 \\
\hline PMSSR & 1,69 & 0,16 & 3,39 & 0,07 & 0,56 & $0,6 \mathrm{~ns}$ \\
\hline$Q$ & 1,57 & 0,19 & 4,69 & 0,03 & 8,95 & 0,0001 \\
\hline
\end{tabular}




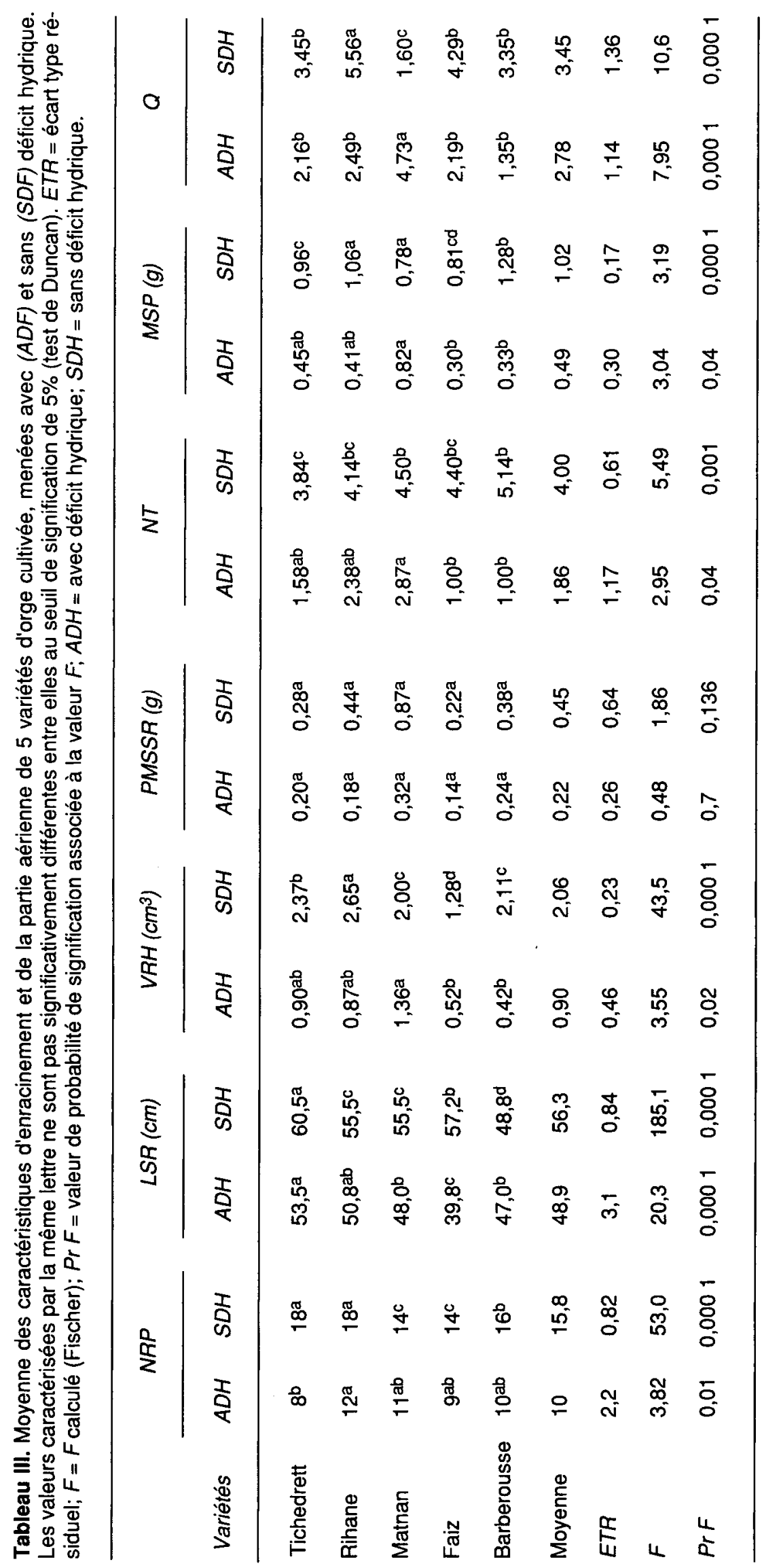




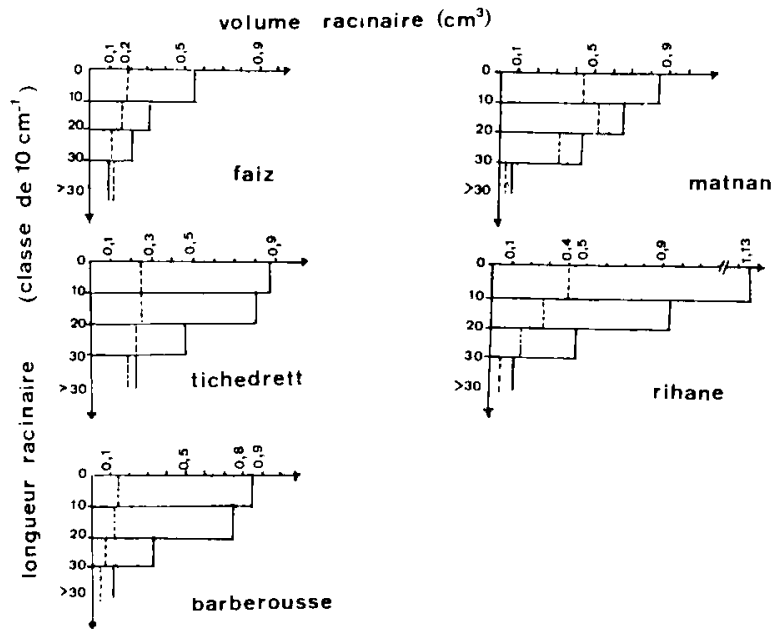

Fig 1. Représentation schématique du volume racinaire en $\mathrm{cm}^{3}$ par longueur racinaire (classe de $10 \mathrm{~cm}^{-1}$ ). (-) sans déficit hydrique, (- - ) avec déficit hydrique.

déficit hydrique : ils sont en effet réduits respectivement de 56,3 et de $51,6 \%$ par rapport au lot témoin (irrigué). Il est à noter que les réductions de la masse de matière sèche et du volume racinaire dans le cas d'un déficit hydrique n'ont pas la même signification, les mesures du volume racinaire tenant compte de la quantité d'eau contenue dans l'échantillon mesuré.

En l'absence de déficit hydrique, ce sont les variétés Rihane et Tichedrett qui possèdent le volume racinaire le plus élevé. On relève un effet marqué du déficit hydrique sur le volume racinaire dans chaque classe de longueur racinaire (tableau III et fig 1); des différences génotypiques peuvent être observées : la variété Matnan maintient une meilleure distribution racinaire que les autres variétés dans les classes de longueur racinaire $A$ et $B$ comprises entre 0 et $20 \mathrm{~cm}$, alors que la variété Tichedrett conserve un volume racinaire plus important à la fois en profondeur (c'est-à-dire dans la classe de longueur racinaire $D>30 \mathrm{~cm}$ ) et dans les premières classes de longueur racinaire comprises entre 0 et 30 $\mathrm{cm}$.

On relève ici que l'étude des caractéristiques racinaires menée dans des pots à contenance limitée a quelque peu biaisé les résultats.

\section{Effet du déficit hydrique \\ sur les caractéristiques de la partie aérienne et sur les relations parties aériennes-racines}

Le déficit hydrique a un effet marqué sur les caractéristiques des parties aériennes étudiées : le nombre de talles et la masse de matière sèche de la partie aérienne sont fortement réduits, le rapport $Q$ (masse aérienne sur masse racinaire)

Tableau IV. Matrice des corrélations entre les différentes caractéristiques d'enracinement et certaines caractéristiques des parties aériennes. ${ }^{*}=$ significatif à $5 \%$; ${ }^{* *}=$ significatif à $1 \%$; ns = non significatif; SDH = sans déficit hydrique; $A D H=$ avec déficit hydrique; $N R P=$ longueur totale du système racinaire; $L S R=$ longueur du système racinaire; $V R H=$ volume total du système racinaire; $P M S S R=$ masse de matière sèche racinaire; $M S P=$ masse de matière sèche des parties aériennes; $N T=$ nombre de talles/plante; $Q=$ masse de matière sèche des parties aériennes sur la masse de matière sèche de la partie racinaire.

\begin{tabular}{|c|c|c|c|c|c|c|c|}
\hline & $N R P$ & LSR & $V R H$ & PMSSR & $M S P$ & $N T$ & $Q$ \\
\hline$N R P$ & $\begin{array}{l}S D H \\
A D H\end{array}$ & $\begin{array}{l}\text { ns } \\
\text { ns }\end{array}$ & $\begin{array}{l}r=0,742^{*} \\
r=4,411^{*}\end{array}$ & $\begin{array}{c}\text { ns } \\
r=0,327^{*}\end{array}$ & $\begin{array}{l}r=0,705^{\star \star} \\
r=0,409^{\star}\end{array}$ & $\begin{array}{l}r=749^{* *} \\
r=0,643^{*}\end{array}$ & $\begin{array}{c}r=0,245^{*} \\
\text { ns }\end{array}$ \\
\hline$L S R$ & $\begin{array}{l}S D H \\
A D H\end{array}$ & & $\begin{array}{l}\text { ns } \\
\text { ns }\end{array}$ & $\begin{array}{l}\text { ns } \\
\text { ns }\end{array}$ & $\begin{array}{c}r=-0,322^{*} \\
\text { ns }\end{array}$ & $\begin{array}{c}r=0,535^{* *} \\
n s\end{array}$ & $\begin{array}{l}\text { ns } \\
\text { ns }\end{array}$ \\
\hline$V R H$ & $\begin{array}{l}S D H \\
A D H\end{array}$ & & & $\begin{array}{c}\text { ns } \\
r=0,408^{*}\end{array}$ & $\begin{array}{l}r=575^{\star \star} \\
r=0,906^{\star \star *}\end{array}$ & $\begin{array}{l}\text { ns } \\
r=0,826^{\star *}\end{array}$ & $\begin{array}{c}\text { ns } \\
r=0,633^{* *}\end{array}$ \\
\hline PMSSR & $\begin{array}{l}S D H \\
A D H\end{array}$ & & & & $\begin{array}{c}\mathrm{ns} \\
r=0,377^{\star *}\end{array}$ & $\begin{array}{c}\mathrm{ns} \\
r=0,351^{*}\end{array}$ & $\begin{array}{l}r=0,994^{* *} \\
r=0,379^{*}\end{array}$ \\
\hline$M S P$ & $\begin{array}{l}S D H \\
A D H\end{array}$ & & & & & $\begin{array}{c}\text { ns } \\
r=0,732^{* *}\end{array}$ & $\begin{array}{c}\text { ns } \\
r=0,440^{*}\end{array}$ \\
\hline$N T$ & $\begin{array}{l}S D H \\
A D H\end{array}$ & & & & & & $\begin{array}{c}\text { ns } \\
r=0,498^{*}\end{array}$ \\
\hline
\end{tabular}


obtenu en conditions de déficit hydrique est inférieur à celui obtenu avec le lot irrigué, sauf dans le cas de la variété Matnan (tableau III).

Les corrélations présentées dans le tableau IV semblent indiquer que les variétés qui possèdent le nombre le plus élevé de racines principales produisent plus de matière sèche dans la partie aérienne, en situation de déficit hydrique $\left(r=0,409^{* *}\right)$ comme en condition irriguée $\left(r=0,705^{*}\right)$. Le nombre de racines principales est étroitement lié à l'aptitude au tallage en condition irriguée $\left(r=0,749^{* *}\right)$ comme en situation de déficit hydrique $\left(r=0,643^{* \star}\right)$. La longueur du système racinaire est toutefois corrélée négativement à la masse de matière sèche de la partie aérienne $\left(r=-0,322^{*}\right)$ en condition irriguée. En situation de déficit hydrique, le volume racinaire est positivement corrélé à la masse de matière sèche du système racinaire $\left(r=0,408^{* \star}\right)$, à la masse de matière sèche de la partie aérienne $\left(r=0,906^{\star *}\right)$ et au nombre de talles par plante $(r$ $\left.=0,826^{* \star}\right)$; en conditions irriguées, le volume racinaire est corrélé à la masse de matière sèche de la partie aérienne $\left(r=0,575^{\star \star}\right.$, fig 4).

\section{Potentiel hydrique foliaire}

Les valeurs du potentiel hydrique $(\psi f)$ (tableau $V$, fig 2) restent élevées et assez voisines pour toutes les variétés pendant les 2 premiers jours du stress. Par contre, au $5^{e}$ jour, des différences variétales apparaissent : on peut en effet distinguer 2 groupes, l'un caractérisé par un potentiel élevé (variétés Tichedrett, Rihane et Matnan) et l'autre par des valeurs très faibles (variétés Barberousse et Faiz).

$P \leq 0,05$

* $P \leq 0,01$

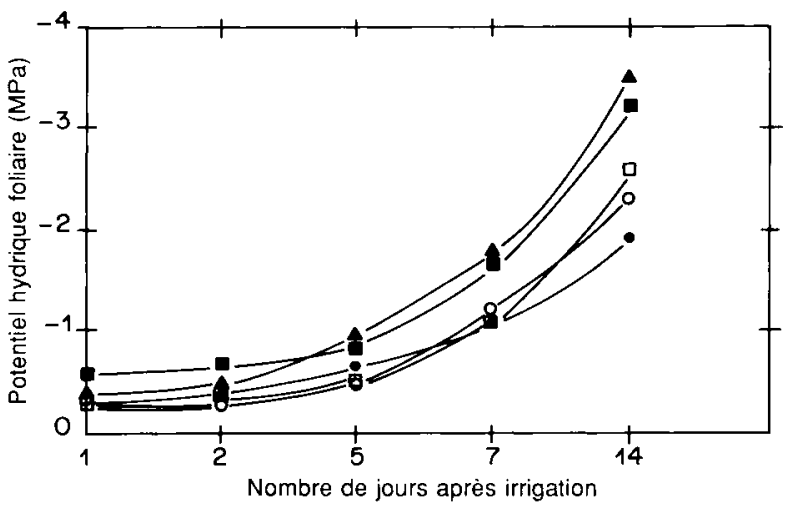

Fig 2. Potentiel hydrique foliaire $(\psi f)$ en fonction du nombre de jours de déficit hydrique, sur 5 variétés d'orge : Barberousse (A), Faiz ( $\square$ ), Matnan (O), Rihane (D), Tichedrett (O), au stade B2 de Jonard.

\section{Teneur en eau de la partie aérienne de la plante}

Les teneurs en eau dans la partie aérienne, exprimées en \% de matière sèche au stade $\mathrm{B} 2$ de Janard (tableau VI), sont significativement plus élevées dans la variété Tichedrett que dans les autres variétés.

\section{Effet du déficit hydrique sur la surface de la dernière feuille (stade B2 de Jonard)}

La mesure de la surface de la dernière feuille développée (S) permet d'apprécier l'influence du déficit hydrique sur la croissance des organes végétatifs aériens.

Les résultats consignés dans le tableau $V$ et la figure 3 montrent l'effet net du déficit hydrique sur la surface foliaire pour toutes les variétés testées; à partir de ces valeurs peut être calculé un indice de sensibilité au déficit hydrique défini par le rapport :

Tableau V. Effets du déficit hydrique sur le potentiel hydrique de la dernière feuille ( $\psi f$, en MPa), au stade B2 de Jonard.

Nombre de jours de stress après irrigation

\begin{tabular}{lccccc}
\cline { 2 - 6 } Variété & 1 & 2 & 5 & 7 & 14 \\
\hline Tichedrett & $-0,45$ & $-0,40$ & $-0,60$ & $-0,80$ & $-1,90$ \\
Rihane & $-0,40$ & $-0,40$ & $-0,40$ & $-0,80$ & $-2,07$ \\
Matnan & $-0,30$ & $-0,40$ & $-0,40$ & $-0,88$ & $-2,32$ \\
Barberousse & $-0,30$ & $-0,45$ & $-0,90$ & $-1,50$ & $-3,50$ \\
Faiz & $-0,50$ & $-0,60$ & $-0,80$ & $-1,40$ & $-3,20$ \\
\hline
\end{tabular}


Tableau VI. Effets du déficit hydrique sur la teneur en eau dans la partie aérienne de la plante (teneur en $\%$ de matière sèche au stade B2 de Jonard). $S D H=$ sans déficit hydrique; $A D H=$ avec déficit hydrique.

\begin{tabular}{lcc}
\hline & \multicolumn{2}{c}{ Traitements } \\
\cline { 2 - 3 } \multicolumn{1}{c}{ Variétés } & $S D H$ & ADH \\
& & \\
Barberousse & $86,55 \pm 0,04$ & $82,7 \pm 0,00$ \\
Faiz & $88,60 \pm 3,74$ & $85,22 \pm 0,14$ \\
Matnan & $87,27 \pm 5,49$ & $86,87 \pm 3,45$ \\
Rihane & $83,74 \pm 2,33$ & $85,10 \pm 0,88$ \\
Tichedrett & $87,90 \pm 1,10$ & $91,11 \pm 1,50$ \\
\hline
\end{tabular}

Tableau VII. Effets du déficit hydrique sur la surface foliaire de la dernière feuille $\left(\mathrm{cm}^{2}\right)$, et indice de sensibilité. $S D H=$ sans déficit hydrique; $A D H=$ avec déficit hydrique.

\begin{tabular}{lccc}
\hline \multirow{2}{*}{ Variétés } & \multicolumn{2}{c}{ Traitements } & \\
\cline { 2 - 3 } & $S D H$ & \multirow{2}{*}{$A D H$} & $S(\%)$ \\
\hline & & & \\
Tichedrett & $39,2 \pm 2,1$ & $19,3 \pm 0,76$ & 50,7 \\
Rihane & $36,7 \pm 0,2$ & $18,7 \pm 1$ & 49,0 \\
Matnan & $24,1 \pm 1,6$ & $8,1 \pm 0,4$ & 66,3 \\
Barberousse & $29,7 \pm 1,4$ & $9,9 \pm 0,5$ & 66,6 \\
Faiz & $19,8 \pm 0,6$ & $6,6 \pm 0,4$ & 66,6 \\
\hline
\end{tabular}

$$
I S=\frac{S A D H-S S D H}{S S D H \times 100}
$$

$S A D H=$ surface de la dernière feuille dans le cas du traitement avec déficit hydrique;
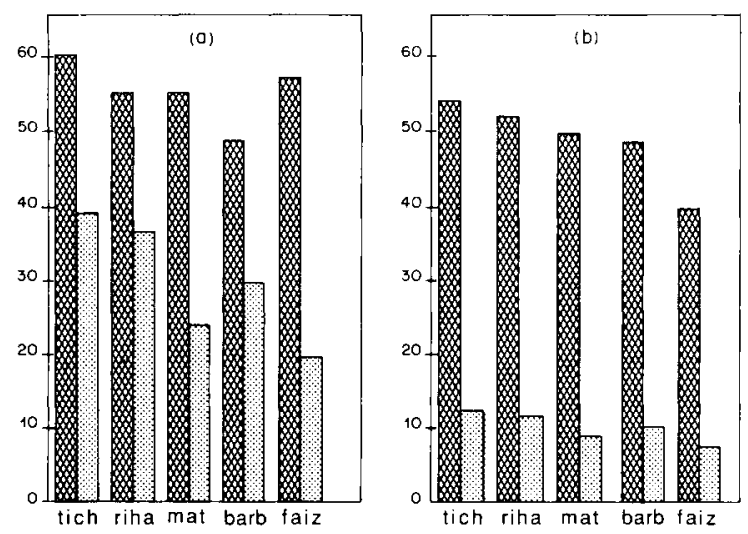

Fig 3. Longueur du système racinaire et surface de la dernière feuille de 5 variétés d'orge cultivées en pots et en serre; en conditions irriguées (a) et en conditions de déficit hydrique (b).
$S S D H=$ surface de la dernière feuille dans le cas du traitement sans déficit hydrique.

Les valeurs obtenues (tableau VII) montrent que le classement par sensibilité décroissante comprend 2 groupes: Faiz, Barberousse, Matnan (sensibilité élevée) et Tichedrett, Rihane (sensibilité plus faible).

\section{DISCUSSION}

L'effet du déficit hydrique sur les caractéristiques des parties aériennes et racinaires est net (tableaux III et VII). Les valeurs de $Q$ (masse aérienne sur masse racinaire) sont faibles pour la plupart des variétés; aussi bien en condition de déficit hydrique qu'en condition irriguée. La variété Matnan fait toutefois exception : le déficit hydrique inhibe plus le développement du système racinaire que la croissance de ses organes aériens.

L'examen des mesures effectuées sur les systèmes racinaires des 5 variétés semble indiquer l'existence d'une relation entre hauteur de paille et développement racinaire : la variété Faiz avec une hauteur moyenne de $70 \mathrm{~cm}$ environ (voir tableau 1), possède un volume (fig 1) et un développement (fig 3) racinaires faibles. Par contre, Tichedrett, qui est une variété à paille haute, possède le système racinaire le plus développé (fig 3).

Ces résultats vont dans le même sens que ceux obtenus par Subbiah et al (1968), Hurd (1974), Bagga et al (1970) sur blé, et ceux de Ali (1977) sur orge, qui démontrent un effet des gènes de nanisme sur le développement racinaire.

Dans le cas de Faiz, il est possible que la réduction de la hauteur de paille soit liée à la précocité de la variété. Par ailleurs, il ressort également de cette étude un effet de la précocité (durée de la période de développement végétatif) sur le développement racinaire : la variété Faiz possède, en effet, un système racinaire moins développé que celui de la variété Tichedrett, plus tartive $(+6 j)$.

Le nombre trop faible de génotypes étudiés (5) ne permet toutefois pas de dégager clairement les rôles respectifs de la hauteur de paille et de la précocité sur le développement racinaire.

L'importance des ramifications racinaires n'a pu être évaluée de manière précise dans le cadre de ce dispositif expérimental : le rapport 


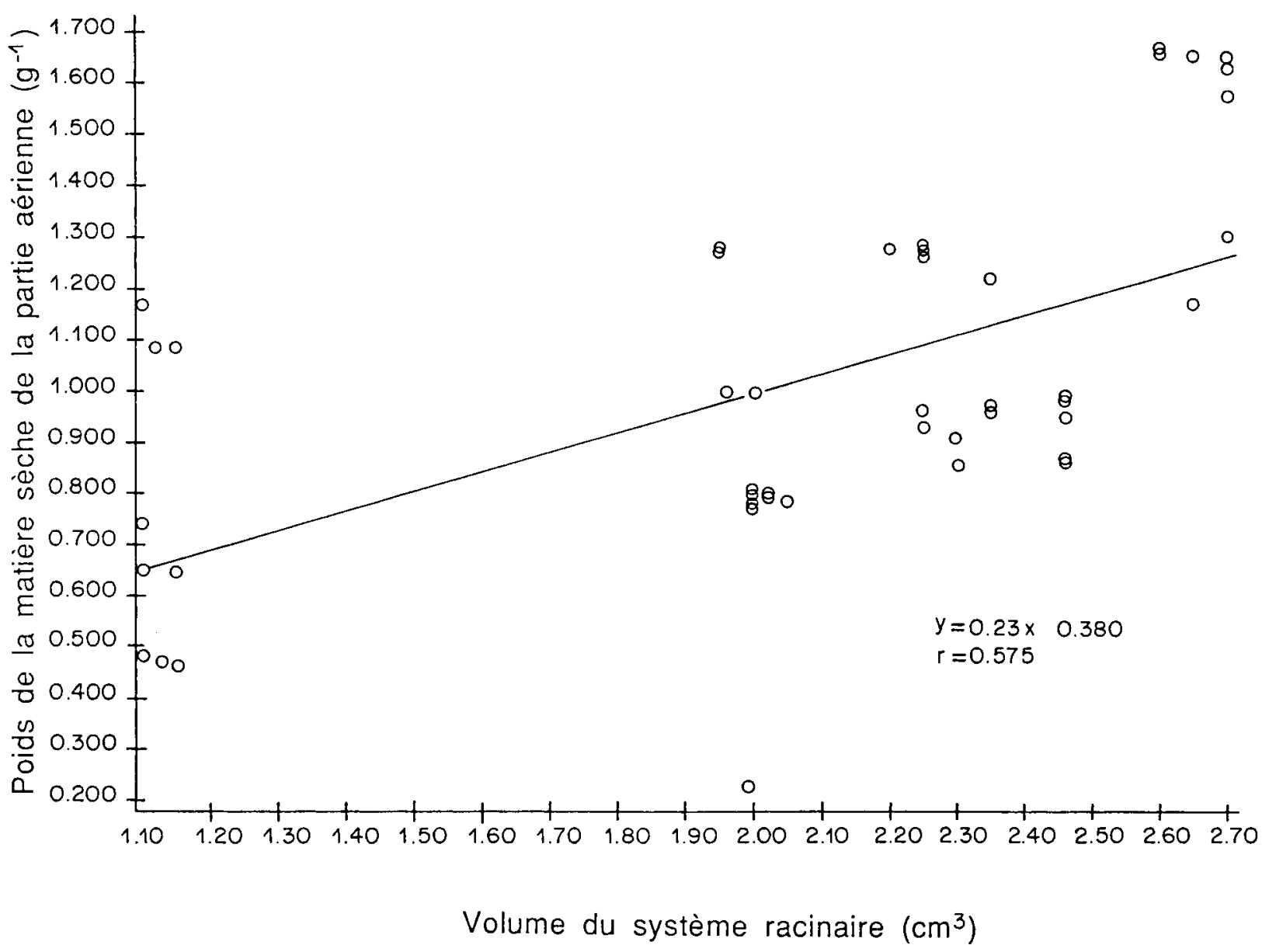

Fig 4. Relation entre le volume racinaire et la masse de matière sèche aérienne, en conditions irriguées.

du poids de la matière sèche racinaire (PMSSR) ou du volume $(V R H)$ de racines, au nombre de racines primaires (NRP), permet toutefois de l'apprécier; le déficit hydrique a un effet plus net sur le volume que sur la masse racinaire (à l'exception de la variété Matnan) : ce résultat vient conforter l'hypothèse émise par Cruiziat (1974), selon laquelle les racines contribuent beaucoup aux pertes en eau subies par une plante durant le dessèchement (linhibition de la croissance des racines est par ailleurs plus forte que celle des organes aériens). Une relation semble donc exister entre les caractéristiques de l'enracinement des divers génotypes et leur tolérance au déficit hydrique. On remarque par exemple que la variété Tichedrett possède un volume racinaire élevé et bien réparti sur le profil (fig 1); nos résultats font également apparaître, chez cette variété, l'existence de racines principales significative- ment plus longues que chez les autres variétés testées; ces caractères permettent à cette variété de mieux explorer le sol en profondeur, surtout en cas de déficit hydrique.

Le rendement en grains de Tichedrett dans les conditions expérimentales de Montpellier (déficit hydrique modéré) reste cependant relativement faible.

Le tableau I permet de comparer les effets, sur le rendement des différentes variétés, d'un déficit hydrique modéré (Montpellier, France, moyenne de 2 ans; Sétif, Algérie 1987-1988) et déficit hydrique intense (Sétif, 1978-1979).

Certes, linterprétation de ces résultats expérimentaux au champ est délicate, car à l'effet hydrique viennent souvent se superposer les conséquences de gelées printanières et de hautes températures en fin de cycle, mais elle permet 
de comparer les mécanismes morphophysiologiques d'adaptation au déficit hydrique étudiés, au comportement agronomique quantifié par la valeur du rapport $N I / I$ (rendement en grain en conditions non irriguées sur rendement en grain en conditions irriguées) (tableau I).

Les variétés Tichedrett, Barberousse, Matnan et Rihane apparaissent comme tolérantes à la sécheresse en raison de mécanismes souvent très différents, bien que le classement de l'indice de sensibilité à la sécheresse NI/I (tableau I) classe Tichedrett et Barberousse dans un premier groupe, Matnan et Rihane dans un groupe intermédiaire et Faiz dans le groupe le moins favorisé par son comportement face au déficit hydrique. La variété Tichedrett, malgré sa tardiveté, a un comportement de résistance au déficit hydrique intéressant, ainsi qu'un développement racinaire efficace. Les variétés Rihane et Matnan allient précocité et résistance à la sécheresse, mais présentent une productivité relativement faible. La variété Barberousse ne présente probablement pas de résistance à la sécheresse aussi nette que les 3 variétés précédentes (potentiel hydrique foliaire plus élevé), mais bénéficie d'une précocité favorable; en outre, elle réagit fort bien aux effets positifs du milieu.

Enfin, Faiz apparaît typiquement comme une variété qui échappe aux effets hydriques grâce à sa grande précocité, mais elle ne semble pas présenter de résistance particulière à la sécheresse (indice de sensibilité à la sécheresse le moins bon). La diversité des stratégies morphophysiologiques d'adaptation doit donc inciter le sélectionneur à mieux définir ses objectifs et les critères de sélection et à améliorer la réponse au déficit hydrique par des combinations judicieuses entre les critères.

\section{CONCLUSION}

Les résultats obtenus dans cette étude confirment les limites de l'expérience menée en pots (technique lourde, imprécise et destructive), déjà mentionnées par plusieurs auteurs (Baldy, 1973; Maertens et Clauzel, 1982; Souty, 1987). Ils nous conduisent à envisager de compléter l'étude de l'enracinement des mêmes génotypes en ayant recours à la technique de la culture aéroponique proposée par Truong et Beunard (1978).

De l'ensemble de ces résultats, une relation semble se dégager entre les caractéristiques d'enracinement et la tolérance au déficit hydri- que des génotypes considérés : 2 types de génotypes tolérant bien la contrainte hydrique, Tichedrett et Rihane (voir tableau I, les rendements obtenus en conditions irriguées et non irriguées à Montpellier) ont tous 2 un volume et une masse racinaire importants (avec un enracinement meilleur pour la variété Tichedrett : plus de $30 \mathrm{~cm}$, et un nombre élevé de racines principales (tableau III); la variété Tichedrett se distingue par ailleurs par un système racinaire profond (tableau III).

Ces caractéristiques racinaires peuvent, en partie, expliquer le fait que ces 2 variétés maintiennent, en cas de déficit hydrique marqué, un potentiel hydrique élevé (tableau $V$ ) et que la croissance de leurs organes végétatifs aériens est relativement peu affectée (tableau III). Les variétés Faiz et Barberousse, au contraire, se caractérisent par un volume racinaire faible, fortement affecté par la contrainte hydrique (fig 1) et par une longueur d'enracinement réduite (tableau III); en cas de dessèchement du sol, leur potentiel hydrique diminue rapidement (tableau $V)$ et la croissance de leurs parties aériennes est très affectée (tableau VII) : l'avantage de la variété Faiz cultivée en zones sèches réside sans doute moins dans ses caractères morphophysiologiques d'adaptation à la sécheresse que dans sa précocité, qui lui permet en particulier d'éviter les déficits hydriques terminaux (de fin de cycle). La variété Matnan occupe, parmi les 5 variétés étudiées, une place intermédiaire : son caractère le plus notable est une croissance racinaire peu affectée par le manque d'eau (fig 1); ce fait pourrait être lié, compte tenu de ce qui a été dit plus haut, à ce que, chez cette variété à faible biomasse aérienne, la redistribution de l'eau contenue dans le réservoir-racines soit plus faible que chez les autres génotypes.

\section{REMERCIEMENTS}

Les auteurs remercient C Baldy, de I'INRA-LECSA de Montpellier (France) pour les remarques constructives et pertinentes apportées en vue de l'amélioration du texte.

\section{RÉFÉRENCES}

Ahmadi N (1983) Variabilité génétique et hérédité de mécanismes de tolérance à la sécheresse chez Oryza sativa L. I. Développement racinaire. Agron Trop 38, 118-122 
Ali MAM (1977) Yield performance of semi-dwart barley (Hordeum vulgare L). Diss Abstr Int B Sci Eng 38,245

Ali-Zade MA, Sultanov JG (1970) Les changements de concentration du suc cellulaire et la capacité de rétention de l'eau par les feuilles de quelques variétés de blé et d'orge, en relation avec leur résistance à la sécheresse (en russe). IzV, Akad Nauk $A z$ SSR, Ser Biol Nauk 5-6, 41-44

Bagga AK, Ruwali KN, Asana RD (1970) Comparison of responses of some Indian and semi-dwarf Mexican wheats to unirrigated cultivation. Indian J Agric Sci $40,421-427$

Baldy C (1973) Progrès récents concernant l'étude du système racinaire du blé (Triticum $\mathrm{sp}$ ) Ann Agron (Paris) 24, 241-276

Bierhuisen JF (1974) Root growth and its environment. Acta Hortic (La Haye) 39, 127-137

Bierhuisen JF (1981) Plant water relationships. Acta Hortic (La Haye) 119, 59-66

Black L (1970) Adventitious roots, tillers and grain yield of spring wheat as influenced by N-P fertilisation. Agron J62, 32-36

Brown SC, Gregory PJ, Wahbi A (1987) Root characteristics and water use in Mediterranean environments. Drought tolerance in winter cereals. Proceedings of the International Workshop 27-31 October 1985, Capri, Italy (JP Srivastava, E Porceddu, E Acevedo, S Varma, eds) John Wiley \& Sons, $387 \mathrm{pp}$

Burch GH, Smith RGC, Mason JC (1978) Agronomic and physiological responses of soybean and sorghum crops to water deficits. II. Crop evaporation, soil water depletion and root distribution. Aust $J$ Plant Physiol 5, 169-177

Cruiziat P (1974) Détermination des pertes en eau subies par les différents organes d'une plante soumise au dessèchement. Ann Agron 25, 539-554

Grignac $P$ (1987) Tolérance au déficit hydrique et aux hautes températures de cultivars de blé. ATP écophysiologie du blé. Rapport intermédiaire campagne 1985, INRA, 279-289

Hurd EH (1974) Phenotype and drought tolerance in wheat. Agric Meteorol 14, 39-55

Hurd EA (1976) Plant breeding for drought resistance in water deficits and plant growth. Academic Press, New York, 4, 317-353

Irivine RB, Harvey BL, Rossnagel BG (1980) Rooting capacity as it relates to soil moisture extraction and osmotic potential of semi-dwarf and normal-statured genotypes of six-row barley (Hordeum vulgare $\mathrm{L}$ ). Can J Plant Sci 60, 242-248

Jonard P, Koller J, Vincent A (1952) Évolution de la tige et de l'épi chez la variété de blé Vilmorin-27 au cours de la période de reproduction. Ann Amelior Plant (Paris) 17, 31-54

Jordan WR, Miller FR (1980) Genetic variability in sorghum root systems : implications of drought resis- tance inadaptation of plants to water and high temperature stress. Wiley Interscience, New York, 383399

Lomas J, Shashoua $Y$ (1973) The effect of rainfall on wheat yield in arid region. In: Plant response to climatic factors. Proceedings of Uppsala Symposium 1970, UNESCO, 531-578

Malet $P$ (1971) Différences de développement radiculaire suivant l'importance des réserves hydriques disponibles en début de végétation sur blé tendre et sur coton. INRA, Doc Tech Tunisie 52, 19 pp

Masle J (1980) L'élaboration du nombre d'épis chez le blé d'hiver. Influence des différentes caractéristiques de la structure du peuplement sur l'utilisation de l'azote et de la lumière. Thèse de DocteurIngénieur INA-PG, Paris, 201 pp

Maertens C, Clauzel Y (1982) Premières observations sur l'utilisation de l'endoscopie dans l'étude de l'enracinement in situ de plantes cultivées. (Sorghum vulgare et Lilium multiflorum). Agronomie 2, 677680

Musick GJ, Fairchild ML, Ferguson VL, Zuber MS (1965) A method of measuring root volume in corn (Zea mays L). Crop Sci 601-602

O'Brien L (1979) Genetic variability of root growth in wheat (Triticum aestivum L). Aust J Agric Res 30 , 587-595

Oertli JJ (1983) Adaptations of plants to limited water availability. In: Nutrient balances and the need for fertilizer in semi-arid and arid zones. Proceedings of the 17th Colloquium of the International Potash Institute, Bern, $394 \mathrm{pp}$

Passioura JB (1981) The collection of water by roots. In: The Physiological and biochimical basis of drought resistance (Paleg, Aspinall, eds) Academic Press, New York, 149-163

Quizenberry JE (1982) Breeding for drought resistance and plant water use efficiency. In: Breeding plants for less favourable environments. Wiley Interscience, New York, 383-399

Renard C, Ndayishimie V (1982) Étude des relations hydriques chez Coffea arabica LT. Comparaison de la presse à membrane et de la chambre à pression pour la mesure du potentiel hydrique foliaire (psi). Café Cacao Thé 26, 27-30

Souty $N$ (1987) Aspect mécanique de la croissance des racines. I. Mesure de la force de pénétration. Agronomie 7, 623-630

Subbiah BV, Katyal JC, Narasimham RL, Dashinamurti $C$ (1968) Preliminary investigation on root distributions of high-yielding wheat varieties. Inst $J$ Appl Radiat 19, 385-390

Sullivan CY (1983) Genetic variability in physiological mechanisms of drought resistance. lowa State $J$ Res 57, 423-439

Truong B, Beunard P (1978) Étude de la croissance racinaire de six variétés de riz pluvial en culture aéroponique. Agron Trop 33, 213-236 